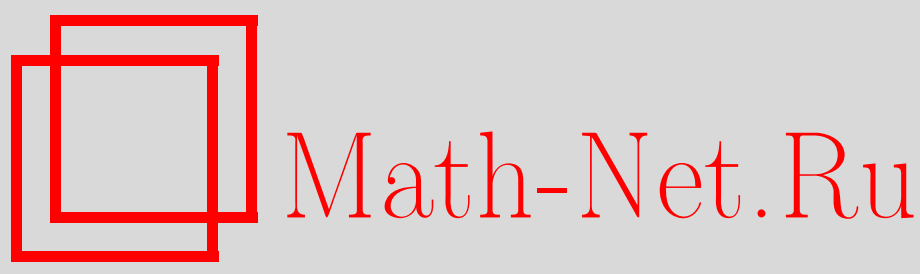

А. А. Махнёв, Д. В. Падучих, О 2-локально графах Зейделя, Изв. РАН. Сер. матем., 1997, том 61, выпуск 4, 67-80

DOI: https://doi.org/10.4213/im136

Использование Общероссийского математического портала Math-Net.Ru подразумевает, что вы прочитали и согласны с пользовательским соглашением

http: //www.mathnet.ru/rus/agreement

Параметры загрузки:

IP : 18.234 .197 .8

26 апреля 2023 г., 16:12:12 
УДК 519.14

\author{
А. А. Махнёв, Д. В. Падучих
}

\title{
О 2-локально графах Зейделя
}

\begin{abstract}
Назовем $i$-окрестностью вершины $a$ графа $\Gamma$ подграф $\Gamma_{i}(a)$, индуцированный $\Gamma$ на множестве всех вершин графа $\Gamma$, которые находятся на расстоянии $i$ от вершины $a$. Пусть $\mathscr{F}$ - некоторый класс графов. Граф Г назовем $i$-локально $\mathscr{F}$-графом, если $\Gamma_{i}(a)$ лежит в $\mathscr{F}$ для любой вершины $a$ графа $\Gamma$. В работе классифицированы связные регулярные графы, в которых 2-окрестности являются графами Зейделя (графом Зейделя называется сильно регулярный графф, имеющий собственное значение, равное -2). Класс графов Зейделя состоит из полных многодольных графов с долями порядка 2 , решетчатых и треугольных графов, а также графов Шрикханде, Чанга, Петерсена, Клебша и Шлефли.

Библиография: 6 наименований.
\end{abstract}

\section{Введение}

В работе рассматриваются неориентированные графы без петель и кратных ребер. Под расстоянием $d(a, b)$ между вершинами $a, b$ графа Г понимается длина кратчайшего пути, соединяюшего $a$ c $b$. Назовем $i$-окрестностью вершины $a$ подграф̆ $\Gamma_{i}(a)$, индуцированный $\Gamma$ на множестве всех вершин графа $\Gamma$, которые находятся на расстоянии $i$ от вершины $a$. Подграф $\Gamma_{1}(a)$ мы будем называть окрестностью вериины $а$ и обозначать через $[a]$. Через $a^{\perp}$ обозначим подграф, индуцированный на $\{a\} \cup[a]$ (на шаре радиуса 1 с центром $a$ ).

Пусть $\mathscr{F}$ - некоторый класс графов. Граф Г назовем $i$-локально $\mathscr{F}$-графом, если $\Gamma_{i}(a)$ лежит в $\mathscr{F}$ для любой вершины $a$ графа $\Gamma$. Если при этом класс $\mathscr{F}$ состоит из графов, изоморфных некоторому графу $\Delta$, то граф Г назовем $i$-локально $\Delta$-графом. При $i=1$ используется выражение: локально граф. Задача описания локально $\mathscr{F}$-графов является классической и решена для различных классов $\mathscr{F}$ (см., например, [1]). Особый интерес эта задача приобрела в свете изучения диаграммных геометрий и их расширений.

Исследование 2-локально $\mathscr{F}$-графов является сравнительно новой задачей. Можно отметить, что изучение 2 -локально $\Delta$-графов диаметра 2 сводится к изучению локально $\bar{\Delta}$-графов. В частности, описание Холлом и Шультом локально котреугольных графов [2] является описанием 2-локально треугольных графов диаметра 2. В статье [3] на некотором шаге возникла задача изучения графов, в которых 2-окрестности оказались прямоугольными решетками, треугольными графами или граффами Шлефли.

Работа выполнена при финансовой поддержке Российского фонда фундаментальных исследований (грант 93-01-01529) и Международного научного фонда совместно с правительством России (грант NMC 300). 
В данной работе изучаются связные регулярные графы, в которых 2-окрестности являются графами Зейделя. Графом Зейделя называется сильно регулярный граф, имеющий собственное значение, равное -2. Класс графов Зейделя обозначим через $\mathscr{S}$. По теореме Зейделя [4] класс $\mathscr{S}$ состоит из полных многодольных графов $K_{n \times 2}$ с $n$ долями порядка 2 , решетчатых и треугольных графов, а также графов Шрикханде, Чанга, Петерсена, Клебша и Шлефли (см. 11 для описания указанных графов).

Валентностью вершины называется число вершин в ее окрестности. Граф Г называется регулярным валентности $k$, если валентность любой вершины из Г равна $k$. Граф Г называется реберно регулярны.м с параметрами $(v, k, \lambda)$, если он имеет $v$ вершин и является регулярным графом валентности $k$, в котором каждое ребро лежит в $\lambda$ треугольниках. Граф $\Gamma$ - вполне регулярный граф с параметрами $(v, k, \lambda, \mu)$, если он реберно регулярен с соответствуюшими параметрами и $[a] \cap[b]$ содержит $\mu$ вершин для любых двух вершин $a, b$, находяшихся на расстоянии 2 в Г. Вполне регулярный граф диаметра 2 называется сильно регулярным графом. Подграф, индуцированньй на $[a] \cap[b]$, назовем $\lambda$-подграфом ( $\mu$-подграфом), если вершины $a, b$ смежны (находятся на расстоянии 2). Граф Г называется редуцированным, если ядро $K(a)=\left\{x \in \Gamma \mid x^{\perp}=a^{\perp}\right\}$ состоит из единственной вершины для любой вершины $a$ графа. Скажем, что граф Г является прямой суммой своих подграфов $\Gamma_{i}$, если подграфы $\bar{\Gamma}_{i}$ являются связными компонентами графа $\bar{\Gamma}$. Лестничным графом называется объединение изолированных ребер. Через $\left(m_{1}, \ldots, m_{n}\right)$ обозначим полньй $n$-дольный граф с долями порядков $m_{1}, \ldots, m_{n}$. Если $m_{1}=\cdots=m_{n}=m$, то граф $\left(m_{1}, \ldots, m_{n}\right)$ обозначается через $K_{n \times m} . \Gamma p a-$ фом Тәйлора называется вполне регулярный графф диаметра 3, в котором каждая вершина лежит в $a^{\perp} \cup b^{\perp}$ для любых двух вершин с $d(a, b)=3$.

Если вершины $u, w$ находятся на расстоянии $i$ в $\Gamma$, то через $b_{i}(u, w)\left(c_{i}(u, w)\right)$ обозначим число вершин в пересечении $\Gamma_{i+1}(u)\left(\Gamma_{i-1}(u)\right)$ с $[w]$. Граф $\Gamma$ диаметра $d$ называется дистанционно регулярным с массивом пересечений $\left\{b_{0}, b_{1}, \ldots, b_{d-1}\right.$; $\left.c_{1}, \ldots, c_{d}\right\}$, если значения $b_{i}(u, w)$ и $c_{i}(u, w)$ не зависят от выбора вершин $u, w$ на расстоянии $i$ в Г. Заметим, что для любого дистанционно регулярного графа $b_{0}-$ это валентность графа, $c_{1}=1$. Дистанционно регулярный граф диаметра $d$ называется антиподальнылм, если отношение - находиться на расстоянии $d$ - является отношением эквивалентности на множестве вершин графа.

ТЕОРемА. Пусть Г - связный регулярный 2-локально $\mathscr{S}$-граф. Тогда либо Г имеет диаметр 3 и является 2-локально $\Delta$-графом Тәйлора, причем граф $\Delta$ изоморфен треугольному графу T(6), $(3 \times 3)$-решетке или графу Шлефли, либо диаметр Г равен 2 и связные компоненты графа $\bar{\Gamma}$ изоморфны следующим графам:

1) регулярный локально лестничный граф;

2) дополнительный граф $\kappa(m+1) \times(m+1)$-решетке или граф Джсонона $J(6,3)$;

3) котреугольный граф $\overline{T(m+2)}$, граф Конвәя-Смита, граф Доро, граф, получаемый из графа изотропных точек симплектического проективного пространства размерности 5 над полем из двух әлементов удалением гиперплоскости или әллиптической квадрики;

4) граф Клебша либо граф Тэйлора, в котором окрестности вершин яв- 
ляются точечными графами обобщенного четырехугольника $G Q(2,4)$, либо сильно регулярный локально $G Q(2,4)$-граф с параметрами $(64,27,10,12)$;

5) дистанционно регулярный граф на 51 вершине с массивом пересечений $\{16,10,1 ; 1,5,16\}$, в котором окрестности вершин изоморфны графу, дополнительному к графу Клебша.

Система инцидентности, состоящая из точек и прямых, называется $\alpha$-частичной геометрией порядка $(s, t)$, если каждая прямая содержит $s+1$ точку, каждая точка лежит на $t+1$ прямой (прямые пересекаются не более чем по одной точке) и для любой точки $a$, не лежащей на прямой $L$, найдется точно $\alpha$ прямых, проходящих через $a$ и пересекающих $L$ (обозначение $p G_{\alpha}(s, t)$ ). Если $\alpha=1$, то геометрия называется обобщенным четырехугольником и обозначается $G Q(s, t)$. Графом Джсонсона $J(n, m)$ называется граф, вершинами которого являются $m$-элементные подмножества данного $n$-элементного множества, и две вершины смежны, если они пересекаются по $(m-1)$-элементному подмножеству. Граф Конвея-Смита на 63 вершинах имеет диаметр 4 и является антиподальным 3-накрытием графа $\overline{T(7)}$. Граф Доро на 65 вершинах является единственным дистанционно регулярным графом с массивом пересечений $\{10,6,4 ; 1,2,5\}$. Он может быть описан как граф коммутирования для класса инволюций групшы $P \Sigma L(2,25)$, не лежащего в коммутанте. Известны существование и единственность графа из п. 5) теоремы $[1$, c. 386$]$.

Класс регулярных локально лестничных графов совпадает с классом реберно регулярных графов, имеюших $\lambda=1$. В классе сильно регулярных графов с $\lambda=1$ известно сушествование точечных графов для $G Q(2, t)$, а также графов с параметрами $(81,20,1,6),(243,22,1,2),(729,55,1,20)$. Отметим здесь проблему Зейделя о существовании сильно регулярного графа с параметрами $(99,14,1,2)$ и статью [6], в которой изучаются сильно регулярные графы с $\lambda=1$, не имеющие двух непересекаюшихся треугольников, соединенных точно двумя ребрами.

\section{§ 1. Редукция к графам диаметра 2}

В этом параграфе мы дадим определения и некоторые свойства графов Зейделя и опишем 2 -локально $\mathscr{S}$-графы диаметра, большего 2.

Прямоугольной решеткой $((m \times n)$-графом) называется граф̆ с множеством вершин $X \times Y$, где $|X|=m,|Y|=n$ и вершины $\left(x_{1}, y_{1}\right),\left(x_{2}, y_{2}\right)$ смежны, только если $x_{1}=x_{2}$ или $y_{1}=y_{2}$. Если $m=n$, то граф называется решеточным $(m \times m)$-графом. Треугольным графом $T(m)$ называется граф с множеством неупорядоченных пар из $X$ в качестве вершин, $|X|=m$ и пары $\{a, b\},\{c, d\}$ смежны тогда и только тогда, когда они имеют общий элемент.

В графе на рис. 1 отождествим вершины $i^{+}, i^{-}(i=1,2, \ldots, 5)$. Затем отождествим вершины 1,5 и $j^{+}, j^{-}(j=6,7,8)$. Полученный сильно регулярный граф с параметрами $(16,6,2,2)$ называется графом Шрикханде. Граф Шрикханде - единственньй нерешеточный граф, имеюший параметры решеточного графа (это параметры $(4 \times 4)$-решетки).

Для нетреугольного графа $\Gamma$, имеюшего параметры треугольного графа, необходимо имеем $m=8$ и $\Gamma$ - один из трех графов Чанга. Для подмножества вершин $X$ графа $\Gamma$ переключением $\Gamma$ относительно $X$ называется граф $\Gamma^{\prime}$ с тем же множеством вершин, причем смежность вершин в $X$ и $\Gamma-X$ остается неизменной, а смежность между вершинами из $X$ и $\Gamma-X$ заменяется на противопо- 


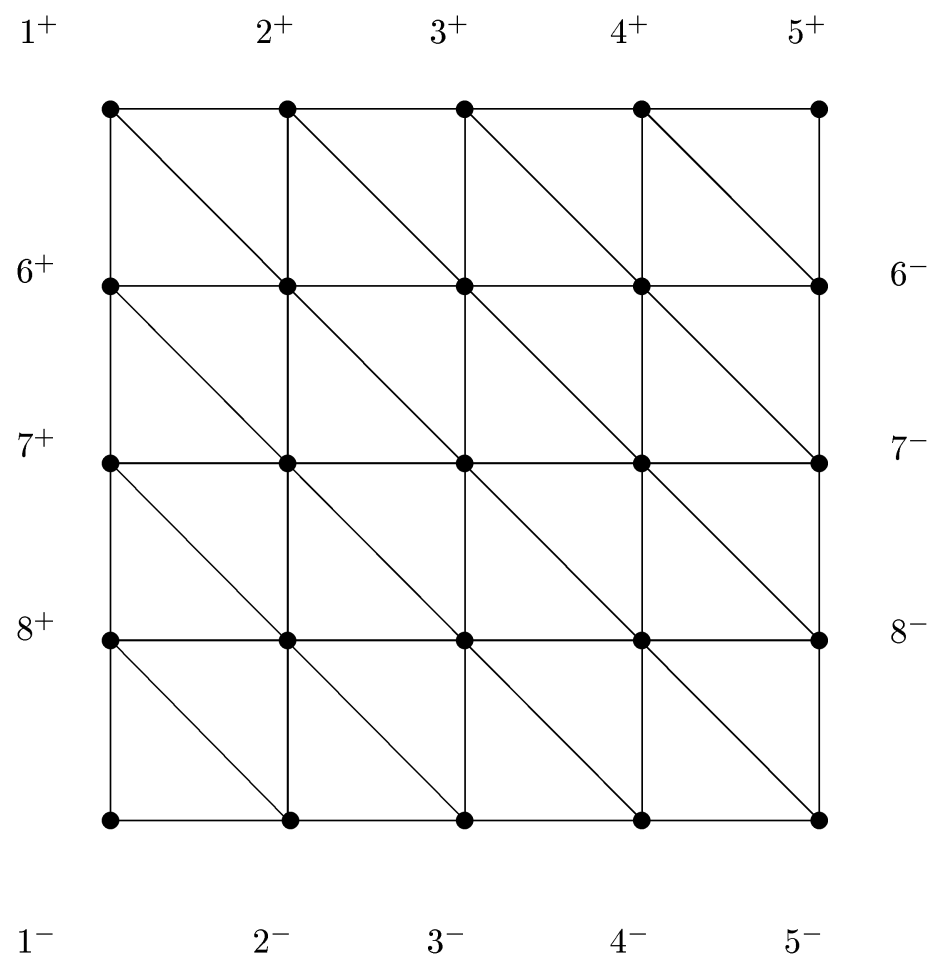

Рис. 1. Граф Шрикханде.

ложную. Теперь графы Чанга $C h_{1}, C h_{2}, C h_{3}$ получаются переключением треугольного графа $T(8)$ относительно множеств $\{15,26,37,48\},\{12,23, \ldots, 78,81\}$, $\{12,23,31 ; 45,56,67,78,84\}$ соответственно.

Граф Петерсена - это сильно регулярный граф с параметрами $(10,3,0,1)$. Он совпадает с графом $\overline{T(5)}$.

Граф Клебиа - это сильно регулярный граф с параметрами $(16,10,6,6)$. Простое описание имеет граф $\Gamma$, дополнительный к графу Клебша. Пусть $a \in \Gamma$ и $[a]=\left\{b_{1}, \ldots, b_{5}\right\}$. Тогда $\Gamma_{2}(a)$ состоит из 10 вершин $c_{i j}$, смежных с $b_{i}, b_{j}$ для различных $i, j \in\{1, \ldots, 5\}$. При этом граф $\Gamma_{2}(a)$ является графом Петерсена. Таким образом, граф Клебша является локально $T(5)$-графом.

Граф Шлефли - это сильно регулярный граф с параметрами $(27,16,10,8)$. Он является дополнительным к точечному графу обобщенного четырехугольника $G Q(2,4)$, причем окрестности вершин в графе Шлефли являются графами Клебша.

В табл. 1 вьписаны параметры графов Зейделя (здесь $r$ - отличное от $k$ неотрицательное собственное значение графа).

ПРЕДЛОЖЕНИЕ 1. Пусть Я્F состоит из регулярных графов диаметра 2. Если связный регулярный редуцированныи 2-локально $\mathscr{F}$-граф Г имеет диаметр, больший 2, то Г - граф Тәйлора и вторые окрестности вершин изоморфнь сильно регулярному графу $\Delta c k(\Delta)=2 \mu(\Delta)$. 
ТАБЛИЦА 1

\begin{tabular}{|c|c|c|c|c|c|c|}
\hline № & Граф & $v$ & $k$ & $\lambda$ & $\mu$ & $r$ \\
\hline 1 & Многодольный & $2 n$ & $2(n-1)$ & $2(n-2)$ & $2(n-1)$ & 0 \\
2 & Треугольный & $n(n-1) / 2$ & $2(n-2)$ & $n-2$ & 4 & $n-4$ \\
3 & Решетчатый & $n^{2}$ & $2(n-1)$ & $n-2$ & 2 & $n-2$ \\
4 & Шрикханде & 16 & 6 & 2 & 2 & 2 \\
5 & Чанга & 28 & 12 & 6 & 4 & 4 \\
6 & Петерсена & 10 & 3 & 0 & 1 & 1 \\
7 & Клебша & 16 & 10 & 6 & 6 & 2 \\
8 & Шлефли & 27 & 16 & 10 & 8 & 4 \\
\hline
\end{tabular}

СлЕДСТВИЕ 1. Пусть Г - сөязный регулярный 2-локально $\mathscr{S}$-граф диаметра, большего 2. Тогда Г- граф Тәйлора, являющийся локально $\Delta$-графом, где $\Delta=T(6), \quad(3 \times 3)$-решетка или граф Шлефли.

ДоКАЗАТЕЛЬСТВО ПРЕДЛОЖЕНИЯ 1 . Пусть Граф̆ Г удовЛетворяет условиям предложения 1. Покажем сначала, что диаметр графа Г равен 3 . Если bcadeгеодезический 4-путь, то $b, e \in \Gamma_{2}(a)$, причем расстояние между $b, e$ в графе $\Gamma_{2}(a)$ не меньше 4. Противоречие.

Пусть $a$ - такая вершина из $\Gamma$, что $\Gamma_{3}(a)$ непусто. Покажем, что любая вершина $x \in \Gamma_{2}(a)$ смежна с любой вершиной $y \in \Gamma_{3}(a)$. В противном случае $\Gamma_{2}(a)$ содержит вершину $z$, находяшуюся на расстоянии 2 от $y$. Противоречие с тем, что $\Gamma_{2}(z)$ содержит $a, y$ и расстояние в $Г$ между $a, y$ равно 3.

Покажем теперь, что $\Gamma_{3}(a)$ является кликой (полным подграфом). В противном случае $\Gamma_{3}(a)$ содержит вершины $b, c c d(b, c)=2$. По предыдушему утверждению вершина $a$ из $\Gamma_{3}(b)$ должна быть смежна $c$. Противоречие.

Итак, $x^{\perp}=y^{\perp}$ для любых вершин $x, y \in \Gamma_{3}(a)$. По редуцированности графа $\left|\Gamma_{3}(a)\right|=1$ и $[a]=\Gamma_{2}(b)$ для $b \in \Gamma_{3}(a)$. Положим $\Delta(x)=\Gamma_{2}(x)$ для $x \in \Gamma$. Число ребер между $[a]$ и $[b]$ равно $|[a]|(|[a]|-k(\Delta(a))-1)$ и равно $|[b]|(|[b]|-k(\Delta(b))-1)$. Отсюда валентность графа $\Delta=\Delta(x)$ не зависит от выбора вершины $x \in \Gamma$. Таким образом, имеем $\mu(a, c)=|\Delta|-k(\Delta)-1$ для любых вершин $a, c$, находяшихся на расстоянии 2 в $Г$. Так как $\Gamma$ - регулярный граф, то для любой вершины $x \in \Gamma$ подграф $\Gamma_{3}(x)$ непуст.

Таким образом, $\Gamma$ - дистанщионно регулярньй граф с массивом пересечений $\{|\Delta|,|\Delta|-k(\Delta)-1,1 ; 1,|\Delta|-k(\Delta)-1,|\Delta|\}$. По теореме Тэйлора-Ливингстона $[1$, теорема 1.5.3] граф $\Delta$ является сильно регулярным с $k(\Delta)=2 \mu(\Delta)$, причем ввиду леммы 1.5.2 $[1]$ и равенства $[a]=\Gamma_{2}(b)$ для $b \in \Gamma_{3}(a)$ каждый графи из $\mathscr{F}$ изоморфен $\Delta$. Предложение 1 доказано.

Для доказательства следствия 1 достаточно заметить, что графы из $\mathscr{S}$ редуцированы, и выбрать в табл. 1 графы $\Delta \mathrm{c} k(\Delta)=2 \mu(\Delta)$. 


\section{§ 2. 2-локально решетчатые графы}

В этом параграфе мы рассмотрим связные регулярные графы диаметра 2, в которых 2-окрестности являются $(m \times m)$-решетками.

Пусть भ्F состоит из связных регулярных графов диаметра $2, \Gamma-v$-вершинный регулярный 2-локально $\mathscr{F}$-граф валентности $k$ и диаметра 2 . Для $a \in \Gamma$ число вершин в $\Gamma_{2}(a)$ не зависит от выбора вершины $a$ и равно $v-k-1$. Положим $\overline{\mathscr{F}}=\{\Lambda \mid \bar{\Lambda} \in \mathscr{F}\}$. Тогда $\bar{\Gamma}$ является локально $\overline{\mathscr{F}}$-графом, и если $\bar{\Gamma}_{i}-$ связные компоненты графа $\bar{\Gamma}$, то граф $Г$ является прямой суммой графов $\Gamma_{i}$. Таким образом, без ограничения обшности мы можем считать граф $\bar{\Gamma}$ связньм. Пусть $a b-$ ребро графа $\bar{\Gamma}$. Тогда граф $\bar{\Gamma}(a) \cap \bar{\Gamma}(b)$ состоит из $(v-k-1)-1-k\left(\Gamma_{2}(a)\right)=(v-k-1)-$ $1-k\left(\Gamma_{2}(b)\right)$ вершин. В силу связности графа $\bar{\Gamma}$ заключаем, что граф $\Gamma_{2}(a)$ имеет валентность, не зависяшую от выбора вершины $a \in \Gamma$.

Пусть граф Г удовлетворяет условиям теоремы и граф $\bar{\Gamma}$ связен. Заметим, что граф̆ Зейделя однозначно определяется своим числом вершин и валентностью, кроме следуюших двух исключений: решеточный $(4 \times 4)$-граф и граф Шрикханде, а также треугольный граф T (8) и три графа Чанга. Но 2-окрестности в графе Шрикханде и $(4 \times 4)$-графе не изоморфны. Значит, если одна 2-окрестность из Г является графом Шрикханде, то Г является 2-локально Шрикханде-графом.

ПРЕДЛОЖЕНИЕ 2. Пусть граф Г является связным регулярным 2-локально $(m \times m)$-графом диаметра $2, m \geqslant 3$, и граф $\bar{\Gamma}$ связен. Тогда Г является $(m+1) \times(m+1)$-решеткой или $m=3$ и $\bar{\Gamma}$ - граф Джсонсона $J(6,3)$.

Пусть граф Г удовлетворяет условиям предложения. Если $m=3$, то граф $\bar{\Gamma}$ является локально $(3 \times 3)$-графом, так как $(3 \times 3)$-решетка самодуальна. В этом случае ввиду [1, с. 258] $\bar{\Gamma}$ является графом Джонсона $J(6,3)$ или графом, дополнительным к $(4 \times 4)$-решетке. Пусть в дальнейшем $m \geqslant 4$.

Лемма 1. Пусть $\Lambda$ является $(m \times m)$-решеткой, $C$-максимальная коклика из $\Lambda$. Тогда $|C|=m$ и для каждой вериины $a \in \Lambda-C$ однозначно определяются вершины $b, c \in C$ такие, что $a \in[b] \cap[c]$.

ДокАЗАТЕЛЬство. Заметим, что для любой вершины $a \in \Lambda$ будем иметь $\Lambda_{2}(a)=(m-1) \times(m-1)$. Утверждение леммы получаем индукцией по $m$. Лемма доказана.

Выберем произвольную вершину $a \in \Gamma$, и пусть $\Lambda=\Gamma_{2}(a), C$ - максимальная коклика из $\Lambda, b, c \in C$. Напомним, что граф Г является кореберно регулярным и $[b] \cap[c]$ содержит $\mu$ вершин. Ясно, что $\mu-2$ из этих вершин лежат в $[a]$. Пусть $\Gamma^{\prime}$ - подграф, индуцированный Г на этих $\mu-2$ вершинах, $\Gamma_{1}=\Gamma-\Gamma^{\prime}$.

Лемма 2. Граф Г является прямой суммой графов $\Gamma^{\prime} u \Gamma_{1}$.

ДокаЗАтельство. Заметим, что любая вершина $d \in C$ смежна со всеми вершинами из $\Gamma^{\prime}$. В противном случае ввиду леммы 1 вершины $b, c$ будут иметь не менее трех общих соседей в $\Gamma_{2}(d)$. Таким образом, подграф $\Gamma^{\prime}$ не зависит от выбора вершин $b, c \in C$.

Возьмем теперь произвольную вершину $e \in \Lambda-C$. По лемме 1 найдутся вершины $x, y \in C$, для которых $e, f$ являются общими соседями в $\Lambda(f$ - некоторая вершина из $\Lambda-C$ ). Заменив в $C$ вершины $x, y$ на $e, f$, получим новую коклику $C^{\prime}$ 
из $\Lambda$. Так как $m \geqslant 4$, то $\left|C \cap C^{\prime}\right| \geqslant 2$, поэтому каждая вершина из $C^{\prime}$ (а значит, и e) смежна со всеми вершинами из $\Gamma^{\prime}$.

Пусть теперь $d \in[a] \cap \Gamma_{1}$. Тогда $\Lambda$ содержит не смежную с $d$ вершину $e . \mathrm{B} \Gamma_{2}(e)$ выберем максимальную коклику $C^{\prime \prime}$, содержашую $a$. Тогда остальные вершины из $C^{\prime \prime}$ лежат в $\Lambda$, поэтому они смежны со всеми вершинами из $\Gamma^{\prime}$. Как показано вьше, каждая вершина из $\Gamma_{2}(e)$ смежна со всеми вершинами из $\Gamma^{\prime}$. Итак, граф $\Gamma$ является прямой суммой графов $\Gamma^{\prime}$ и $\Gamma_{1}$. Лемма доказана.

Теперь можно считать, что $\Gamma=\Gamma_{1}$.

ЛЕмма 3. Граф Г является $(m+1) \times(m+1)$-решеткой.

ДокАЗАТЕЛЬСТво. Заметим, что граф Г кореберно регулярен с $\mu=2$. Далее, $\mu$-подграфы $[a] \cap[b]$ и $[a] \cap[c]$ не пересекаются для любых различных вершин $b, c \in C$, поэтому для валентности $k$ графа $\Gamma$ имеем неравенство $k \geqslant 2 n$. С другой стороны, каждая вершина из $[a]$ смежна с некоторой вершиной коклики $C$. Действительно, если вершина $d \in[a]$ не смежна с вершиной $e \in C$, то, выбрав в $\Gamma_{2}(e)$ коклику $C^{\prime}=(C-\{e\}) \cup\{a\}$, мы можем применить к ней лемму 1.

Итак, $k=2 n$. Теперь число вершин в графе $\Gamma$ равно $(n+1)^{2}$. Покажем, что граф $\Gamma$ реберно регулярен. Пусть $d \in[a]$. Тогда вершина $d$ смежна с единственной вершиной $e \in C$. Легко понять, что $d$ смежна в $\Gamma_{2}(a)$ только с соседями вершины $e$. В самом деле, допустим, что $d$ смежна в $\Gamma_{2}(a)$ с вершиной $x$, не смежной с $e$. По лемме 1 найдутся вершины $b, c \in C$, для которых $[b] \cap[c]=\{x, y\}$. Заменив в $C$ вершины $b, c$ на $x, y$, получим коклику $C^{\prime}$, содержашую две вершины, смежные $\mathrm{c} d$. Противоречие с леммой 1.

Аналогично найдем, что для каждой отличной от $e$ вершины $b \in C$ вершина $d$ смежна точно с одной вершиной из $[b] \cap[e]$. В противном случае достаточно заменить в $C$ вершины $b, e$ на $[b] \cap[e]$, и в полученной коклике $d$ будет смежна с единственной вершиной. Таким образом, вершина $d$ имеет в $\Gamma_{2}(a)$ точно $n$ coседей. Отсюда $[a] \cap[d]$ содержит точно $n-1$ вершин. Итак, $\Gamma$ - сильно регулярный граф с параметрами $\left((n+1)^{2}, 2 n, n-1,2\right)$ и в силу теоремы Зейделя является $(n+1) \times(n+1)$-решеткой. Предложение 2 доказано.

\section{§ 3. Треугольные графы и графы Чанга}

В этом параграфе мы рассмотрим связные регулярные графы диаметра 2, в которых 2-окрестности являются графами Чанга или треугольными графами.

ПРЕДЛОЖЕНИЕ 3. Пусть Г - связный регулярный граф диаметра 2, в котором 2-окрестности являются треугольными графами $T(m), m \geqslant 5$, или графами Чанга. Если граф $\bar{\Gamma}$ связен, то граф Г является либо треугольным графом $T(m+2)$, либо $m=5$ и $\bar{\Gamma}$ является графом Конвея-Смита на 63 вершинах или графом Доро на 65 вериинах, либо $m=6$ и $\bar{\Gamma}$ получается из графа изотропных точек симплектического проективного пространства размерности 5 над полем из двух әлементов удалением гиперплоскости или әллиптической квадрики.

Пусть граф̆ Г удовлетворяет условиям предложения 3. Заметим, что в графах Чанга нет 2-окрестностей вершин, изоморфных треугольному графу. Отсюда либо все 2-окрестности вершин из Г являются треугольными графами, либо все они 
являются графами Чанга. В первом случае по теореме Холла-Шульта [2] выполняется заключение предложения 3. Пусть до конща параграфа все 2-окрестности в графе $Г$ являются графами Чанга. Для несмежных вершин $a, b \in \Gamma$ положим $\Delta(a, b)=\Gamma_{2}(a) \cap \Gamma_{2}(b)$.

ЛЕмма 4. Пусть $\Lambda$ - граф Чанга $C h_{1}$, полученный переключением графа $T(8)$ относительно множества $X=\{15,26,37,48\}$. Тогда граф $\Lambda_{2}(15)$ является обгединением 3 -коклики $X_{0}=\{26,37,48\} u(2 \times 6)$-решетки $\{12,13,14,16$, $17,18 ; 52,53,54,56,57,58\}$, причем вершина из $X_{0}$ смежна с вершиной из решетки, если они не содержат общих әлементов из $\{1, \ldots, 8\}$. Далее, если 2-окрестность некоторой вериинь $x$ в графе Чанга содержит 6-клику, то этот граф получен переключением графа $T(8)$ относительно множества вериин $X=\{15,26,37,48\}$ и $x \in X$.

ДокАЗАТЕльство. Первое утверждение леммы тривиально. Второе получается просмотром 2-окрестностей вершин в граффах Чанга.

Лемма 5. Пусть $a, b$ - несмежные вериинь в графе Чанга $\Lambda$ и $\Lambda_{2}(a) \cap$ $\Lambda_{2}(b)$ содержит 5-клику L. Тогда точно одна из вериин в $a, b$ лежит $в$ переключательном множестве $X$.

ДокаЗАТЕЛЬСтво. Пусть $\Lambda=C h_{1}$. Тогда 2-окрестности вершин, не лежаших в переключательном множестве $X=\{15,26,37,48\}$, изоморфны. Далее, $\Lambda_{2}(12)=(T\{34 \ldots 8\}-\{37,48\}) \cup\{15,26\}$ и все вершины произвольной 5 -клики $L$ из $\Lambda_{2}(12)$ содержат либо 5 , либо 6 . В этом случае только вершина из $\{15,26\}$ не смежна с вершинами из $L$.

Пусть $\Lambda=C h_{2}$. Тогда имеется 4 типа 2-окрестностей вершин, не лежаших в переключательном множестве $X=\{12,23, \ldots, 78,81\}$. Далее, $\Lambda_{2}(13)=(T\{24 \ldots 8\}-$ $\{45,56,67,78\}) \cup\{12,23,34,81\}$ и единственная 5 -клика $L$ из $\Lambda_{2}(13)$ совпадает с $\{24,25,26,27,28\}$. Но в этом случае только вершины 12,23 не смежны с вершинами из $\Lambda_{2}(13)$ и утверждение леммы вьполняется.

Для вершин 14, 15, 16 их 2-окрестности не содержат 5-клик.

Пусть $\Lambda=C h_{3}$. Тогда имеется 3 типа 2-окрестностей вершин, не лежаших в переключательном множестве $X=\{12,23,13 ; 45,56,67,78,84\}$. Для вершины 14 ее 2 -окрестность не содержит 5-клик. Далее, $\Lambda_{2}(46)=(T\{123578\}-\{12,23,13 ; 78\}) \cup$ $\{45,56,67,84\}$ и единственная 5-клика $L$ из $\Lambda_{2}(13)$ совпадает с $\{51,52,53,57,58\}$. Но в этом случае только вершины 45,56 не смежны с вершинами из $\Lambda_{2}(13)$ и утверждение леммы выполняется.

Аналогично рассматривается $\Lambda_{2}(47)$. Лемма доказана.

ЛЕмма 6. Не существуют связные регулярные графы, в которых 2-окрестности являются графами Чанга.

ДоказАТЕльство. Пусть $a \in \Gamma$ и граф $\Lambda=\Gamma_{2}(a)$ является графом Чанга. Если $\Lambda$ является графом $C h_{1}$, полученным переключением графа $T(8)$ относительно $X=\{15,26,37,48\}$, то положим $b=15, c=12$. Тогда граф $\Delta(a, b)-c^{\perp}$ является полным графом на шести вершинах с одним удаленным ребром. Этот же граф получается еще в одном случае: $\Lambda$ является графом Чанга $C h_{2}$, полученным переключением $T(8)$ относительно $X=\{12,23, \ldots, 78,81\}, b=13, c=34$. Если 
же $\Lambda$ является графом Чанга $C h_{3}$, полученным переключением $T(8)$ относительно $X=\{12,23,13 ; 45,56,67,78,84\}$, то пусть $b=45, c=46$.

Тогда подграф $\Delta(a, b)-c^{\perp}$ содержит 5 -клику. По строению графа $\Delta(a, c)$ заключаем, что $a$ не лежит в переключательном множестве для граффа $\Gamma_{2}(c)$. Ввиду леммы 5 переключательное множество для графа $\Gamma_{2}(c)$ содержит вершину $b$. Но теперь переключательное множество для графа $\Gamma_{2}(b)$ содержит вершины $a, c$. Противоречие с леммой 5 . Лемма 6 , а вместе с ней и предложение 4 доказаны.

\section{§ 4. Графы Петерсена, Шлефли и Шрикханде}

В этом параграфе мы рассмотрим связные регулярные графы диаметра 2, в которых 2-окрестности являются графами Петерсена, Шрикханде или графами Шлефли.

ПРЕДЛОЖЕНИЕ 4. Пусть Г - связный регулярный граф диаметра 2 с 2-окрестностями вершин, изоморфными графам Петерсена, Шлефли или Шрикханде. Тогда связные компоненты графа $\bar{\Gamma}$ изоморфны графу Клебиа или локально $G Q(2,4)$-графу Тәйлора, или сильно регулярному локально $G Q(2,4)$-графу с параметрами $(64,27,10,12)$.

ДоКАЗАТЕЛЬСТво. Пусть граф Г удовлетворяет условиям предложения 4. Сначала допустим, что 2-окрестности вершин из $Г$ изоморфны графу Петерсена. Тогда граф $\bar{\Gamma}$ является локально $T(5)$-графом. Без ограничения обшности можно считать, что граф $\bar{\Gamma}$ связен. Заметим, что $\mu$-подграфы в локально $T(m)$-графе являются локально четырехугольниками, поэтому $\mu$-подграфы являются объединениями изолированных октаэдров, число которых не превосходит $m / 4$. Таким образом, $\bar{\Gamma}$ является сильно регулярным графом с параметрами $(16,10,6,6)$ и совпадает с графом Клебша.

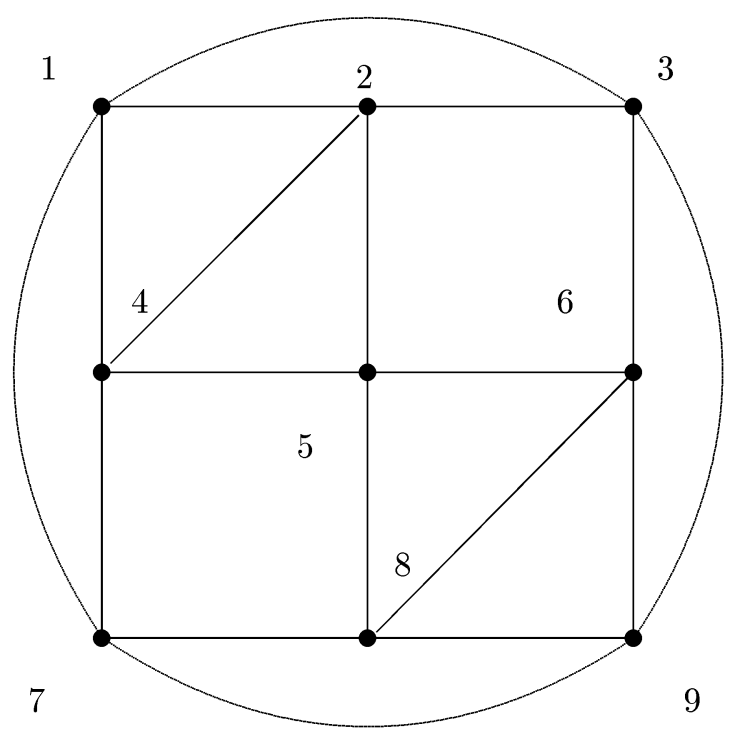

Рис. 2. Граф $\Delta_{0}$ 
Пусть теперь Г является графом, в котором 2-окрестности изоморфны графу Шлефли. Тогда граф $\bar{\Gamma}$ является локально $G Q(2,4)$-графом. Из [5] следует справедливость предложения 4 в рассматриваемом случае.

Пусть, наконец, Г является графом, в котором 2-окрестности изоморфны графу Шрикханде. Для вершины $a \in \Gamma$ пусть $\Delta=\Gamma_{2}(a)$. Тогда граф $\Delta_{0}=\Delta \cap \Gamma_{2}(b)$ для $b \in \Delta$ изображен на рис. 2 .

Пусть $c \in[a] \cap \Gamma_{2}(b)$. По строению графа $\Gamma_{2}(b)$ вершина $c$ смежна в $\Delta_{0}$ с одним из 2-путей: $258,456,239,136,479,178$. Без ограничения обшности вершина $c$ смежна с вершинами $2,5,8$. Далее, $\Delta(b)$ - шестиугольник, и точно три вершины $3,5,7$ смежны в нем с парами антиподальных вершин, а остальные вершины из $\Delta_{0}$ смежны с ребрами шестиугольника.

Пусть вершина 5 смежна с парой антиподальных вершин $5^{+}, 5^{-}$в шестиугольнике $\Delta(b)$. Очевидно, что $c \in \Gamma_{2}(4)$. Так как вершины 2,5 не лежат в $\Gamma_{2}(4)$, то $c$ смежна еще с двумя вершинами $d, e \in \Delta(b)$. Вершины $d, e, 8$ образуют 2-путь в $\Gamma_{2}(4) \cap \Delta$, причем вершина 8 является крайней в этом 2-пути, так как она смежна с ребром $e f$ графа $\Delta(b)$.

Напомним, что в графе Шрикханде $\mu=2$, поэтому вершины 2,8 имеют в $\Delta(b)$ единственного обшего соседа. Аналогично, $\lambda=2$ в графе $\Delta$, поэтому пары вершин 5,8 и 5,2 имеют по одному общему соседу в $\Delta(b)$. С другой стороны, вершины $5^{+}, 5^{-}$находятся на расстоянии 3 в $\Delta(b)$, следовательно, тройка вершин $2,5,8$ имеет в $\Delta(b)$ общего соседа. По симметрии то же самое верно для тройки вершин $4,5,6$.

Пусть вершина 4 смежна с ребром $x y$ граффа $\Delta(b)$, а вершина 6 - с ребром $y z$ (см. рис. 3). Покажем, что вершина 2 смежна с ребром $d e$.
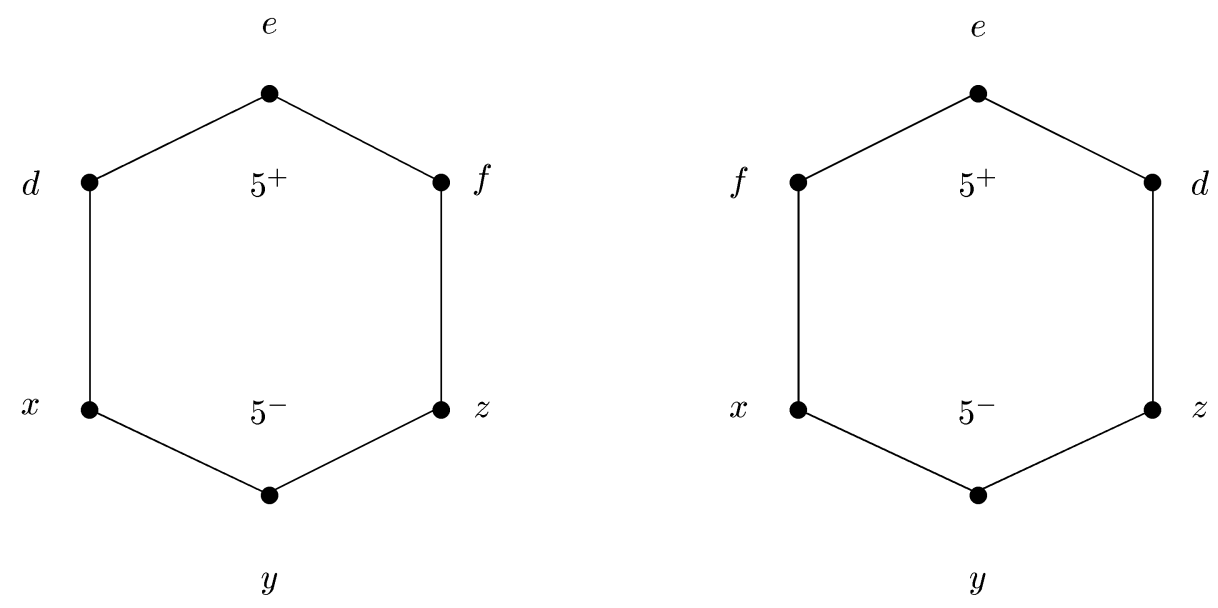

Рис. 3. Граф $\Delta(b)$

В противном случае вершина 2 смежна с ребром $f w$ графа $\Delta(b)$ (см. рис. 4$)$. Рассмотрим подграфф $\Gamma_{2}(6)$. Из рассуждений, проведенных для вершины 4 , следует, что вершина $c$ смежна с ребром в 2 -пути $\Gamma_{2}(6) \cap \Delta(b)$. Противоречие с тем, что вершина $c$ смежна с $e$ и не смежна с $f$. 


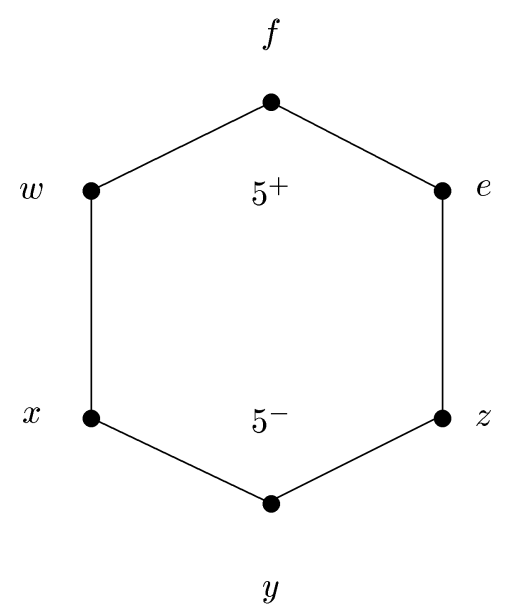

Рис. 4. Граф $\Delta(b)$

Таким образом, мы показали, что вершина 5 смежна с $e$. Но тогда одна из вершин 3,7 не смежна ни с $e$ ни с $d$. Пусть для определенности это будет вершина 3 . В этом случае вершина $c$ смежна с вершинами $5,8, e, d$ в $\Gamma_{2}(3) \cap \Delta(b)$. Противоречие. Предложение 4 доказано.

\section{§5. Граф Клебша}

В этом параграфе мы рассмотрим связные регулярные графы диаметра 2, в которых 2-окрестности являются графами Клебша.

ПРЕДЛОЖЕНИЕ 5. Связный регулярный граф диаметра 2 с 2-окрестностями вершин, изоморфнылми графу Клебша, является прямой суммой графов, дополнительных к дистанционно регулярному графу с массивом пересечений $\{16,10,1 ; 1,5,16\}$.

Заметим, что дополнительный граф к графу Клебша является сильно регулярным с параметрами $(16,5,0,2)$. Мы докажем, что для сильно регулярного графа $\Delta$ с параметрами $(16,5,0,2)$ связный локально $\Delta$-граф $\Gamma$ является дистанционно регулярным с массивом пересечений $\{16,10,1 ; 1,5,16\}$.

ЛЕмма 7. Пусть $\Lambda$ является индуцированным п-угольником из $\Delta$. Тогда $n \leqslant 6$ и выполняются следующие утверждения:

а) если $n=4$, то каждая вершина из $\Lambda$ смежна с 3-кокликой вне $\Lambda$, каждая верична вне $\Lambda$ смежна с единственной вершиной из $\Lambda$;

b) еслип $=5$, то пять вершин вне $\Lambda$ смежны с парами несмежных вершин из $\Lambda$, еще пять вершин вне $\Lambda$ смежны с единственными вершинами из $\Lambda$ и точно одна вершина вне $\Lambda$ не смежна с вершинами из $\Lambda$;

с) если $n=6$, то две вершины вне $\Lambda$ смежны с 3-кокликами из $\Lambda$, три пары вериин вне $\Lambda$ смежны с парами антиподальных вериин в $\Lambda$, точно две вершины вне $\Lambda$ не смежны с вериинами из $\Lambda$. 


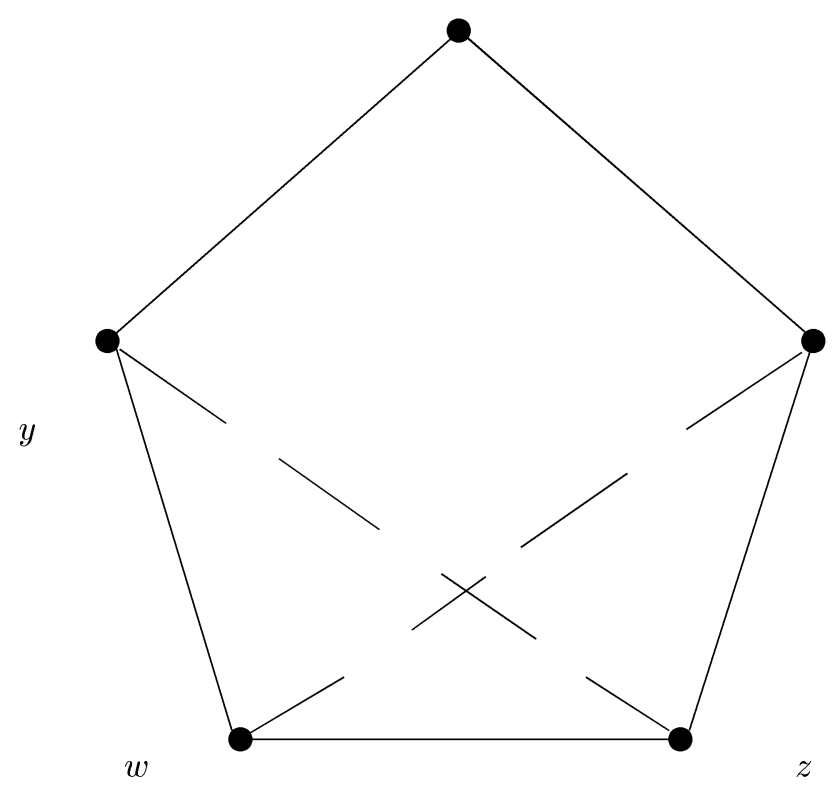

Рис. 5. Граф $[a] \cap[x]$

ДоКАЗАТЕЛЬСТво. Для $n=4,5,6$ утверждения леммы проверяются непосредственным подсчетом. Пусть $a b c-2$-путь из $\Lambda, d$ - отличная от $b$ вершина из $[a] \cap[c]$. По утверждению а) леммы $n-5$ вершин из $\Lambda-\left(a^{\perp} \cup c^{\perp}\right)$ смежны с $d$, поэтому в случае $n>6$ граф̆ $\Delta$ содержит треугольник.

Лемма 8. Для любых вершин $a, b$, находящихся на расстоянии 2 в Г, подграф $[a] \cap[b]$ является четырех- или пятиугольником.

ДокАЗАТЕЛЬСтво. Валентность любой вершины $c$ в графе $[a] \cap[b]$ равна 2 , поэтому граф $[a] \cap[b]$ является объединением изолированных $n$-угольников. В силу леммы 7 этот граф связен и $n=4,5,6$.

Покажем, что случай $n=6$ невозможен. Пусть $x, y, z$-коклика из $[a] \cap[b]$. По утверждению с) леммы 7 граф [b] содержит единственную вершину $c$, смежную $c$ вершинами $x, y, z$. Тогда граф $[a] \cap[c]$ также является шестиугольником. Противоречие с тем, что граф $\Delta$ не содержит пары шестиугольников, пересекающихся по 3-коклике. Лемма доказана.

Введем следующие обозначения:

$Q(a)=\{x \in \Gamma|4=|[a] \cap[x] \mid\}$ - подграф "четырехугольных" относительно $a$ вершин;

$P(a)=\{x \in \Gamma|5=|[a] \cap[x] \mid\}-$ подграф̆ "пятиугольных" относительно $a$ вершин.

ЛЕмма 9. Выполняются следующие утверждения:

а) если $\Gamma_{3}\left(\right.$ (а) не пусто, то граф $\Gamma_{3}($ a) является кокликой;

b) диаметр графа Г не больше 3 ;

c) любая вершина из $P(a)$ смежна с единственной вершиной из $\Gamma_{3}(a)$. 


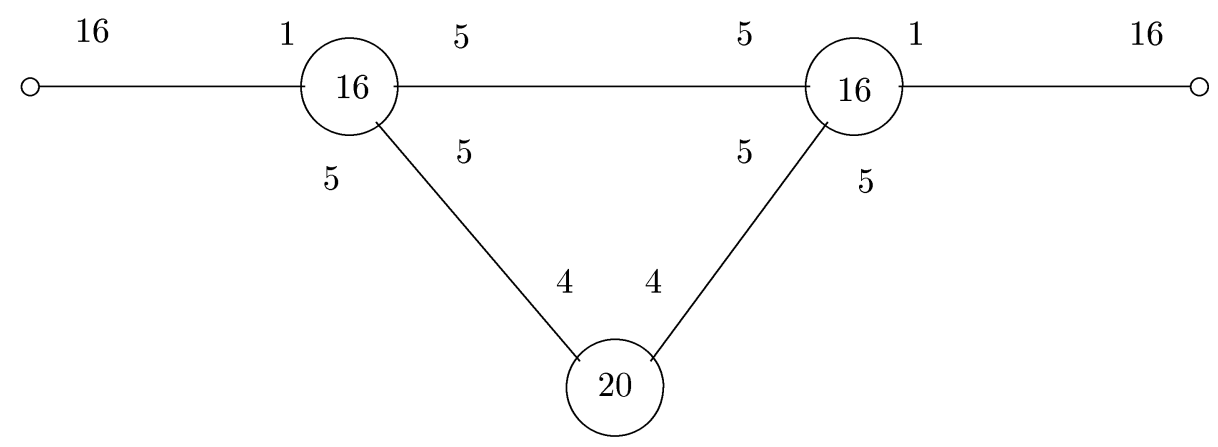

8

Рис. 6. Диаграмма граффа относительно произвольной вершины

ДокаЗАТЕльство. Заметим сначала, что если $b \in Q(a)$, то по лемме 7 граф $[b]$ содержится в $\Gamma_{2}(a)$. Отсюда $[c] \cap \Gamma_{2}(a) \subset P(a)$ для любой вершины $c \in \Gamma_{3}(a)$. По лемме 7 вершина из $P(a)$ смежна не более чем с одной вершиной из $\Gamma_{3}(a)$. Если вершина $c \in \Gamma_{3}(a)$ смежна с вершиной $d$ вне $\Gamma_{2}(a)$, то $[c] \cap[e]-$ пятиугольник, не пересекающий $[d]$ для любой вершины $e \in \Gamma_{2}(c) \cap[a]$. Из пяти вершин в $[c] \cap[d]$ не менее четырех лежат в $\Gamma_{2}(e)$. Противоречие с леммой 7 . Утверждения $\left.\left.\mathrm{a}\right), \mathrm{b}\right)$ леммы 9 доказаны.

Допустим, что $b \in P(a)$ и $[b]$ не пересекает $\Gamma_{3}(a)$. Вершина $c \in[b]$, не смежная с вершинами пятиугольника $[a] \cap[b]$, смежна в $[b]$ с пятью вершинами, каждая из которых смежна с парой вершин из $[a] \cap[b]$. По предположению $c \in \Gamma_{2}(a)$, причем подграфы $[a] \cap[b]$ и $[a] \cap[c]$ не пересекаются. По лемме $7 c \in P(a)$.

Как показано выше, вершины из $[b] \cap[c]$ образуют коклику, и каждая из них смежна с коребром из $[a] \cap[b]$ и с коребром из $[a] \cap[c]$. Отсюда $[b] \cap[c] \subset P(a)$, иначе некоторое коребро из $[a] \cap[b]$ смежно с коребром из $[a] \cap[c]$ и с вершиной из $[a] \cap[b]$. Противоречие.

Для вершины $x \in[b] \cap[c]$ расположение двух коребер в пятиугольнике $[a] \cap[x]$ определяется однозначно (см. рис. 5). Таким образом, если вершина $x$ смежна с вершинами $y, z \in[a] \cap[b]$, то она смежна с вершиной $w \in[a] \cap[c]$, причем $w \in[y] \cap[z]$. Отсюда следует, что пятиугольник $[a] \cap[c]$ образован соседями коребер пятиугольника $[a] \cap[b]$ и, следовательно, должен быть кокликой. Противоречие.

Лемма 10. Число вершин в $\Gamma_{3}($ (а) равно 1 или 2 и не зависит от вььбора вериины $а$.

ДокАЗАТЕЛЬСтво. По лемме $9|P(a)|=16\left|\Gamma_{3}(a)\right|$. Число ребер между $[a]$ и $\Gamma_{2}(a)$ равно $5|P(a)|+4|Q(a)|=160$, поэтому $|Q(a)|=40-20\left|\Gamma_{3}(a)\right|$, в частности, $\left|\Gamma_{3}(a)\right| \leqslant 2$. Подсчитав число вершин в графе $\Gamma$ для каждого из возможных значений $\left|\Gamma_{3}(a)\right|$, получим $57,54,51$ соответственно. Следовательно, значение $\left|\Gamma_{3}(a)\right|$ не зависит от выбора вершины $a$.

Допустим, что $\left|\Gamma_{3}(a)\right|=0$. Тогда $Г$ является сильно регулярным графом с параметрами $(57,16,5,4)$. Но этот граф не удовлетворяет условию целочисленности.

ЛЕмма 11. Число вершин в $\Gamma_{3}(a)$ равно 2. 
ДоКАЗАТЕЛЬСТво. Пусть $\left|\Gamma_{3}(a)\right|=1$. Для произвольной вершины $x \in \Gamma$ через $x^{*}$ обозначим единственную вершину из $\Gamma_{3}(x)$. Тогда $P\left(a^{*}\right)=[a], Q(a)=Q\left(a^{*}\right)$ и граф Г имеет диаграмму относительно любой вершины (см. рис. 6$)$.

Если $x \in Q(a)$, то вершина $x$ смежна с четырехугольниками из $[a]$ и $\left[a^{*}\right]$, образуюшими куб в силу леммы 7 . Нетрудно показать, что тогда $[x] \cap Q(a)$ также является кубом и $Q(a)$ - локально кубический граф на 20 вершинах. Повторив рассуждения из доказательства леммы 8 , получим, что $\mu$-подграфы в $Q(a)$ - это либо четырехугольники, либо коклики из не более чем двух вершин.

Пусть $x \in Q(a)$. Тогда $x^{*} \in Q(a)$ и $\mu$-граф $[x] \cap[y]$ содержит не более двух вершин из $Q(a)$ для восьми вершин $y \in Q(a) \cap\left[x^{*}\right]$. Таким образом, $\mu$-граб $[x] \cap[y]$ содержит четыре вершины из $Q(a)$ для не более двух вершин $y \in Q(a)$. Противоречие с тем, что число ребер между окрестностью $x$ в графе $Q(a)$ и 2-окрестностью $x$ в этом же графе равно 32 . Лемма доказана.

Таким образом, Г - дистанционно регулярный граф с требуемым массивом пересечений. Предложение 5 , а вместе с ним и теорема доказаны.

\section{Список литературы}

1. Brouwer A.E., Cohen A.M., Neumaier A. Distance-Regular Graphs. Berlin etc: Springer-Verlag, 1989.

2. Hall J. I., Shult E. E. Locally cotriangular graphs // Geom. Dedic. 1985. V. 18. № 1. P. 113-159.

3. Кабанов В.В., Махнёв А.А. Кореберно регулярные графы, в которых антиокрестности вершин кореберно регулярны: Тез. докл. // III Международная конференция по алгебре. Красноярск, 1993. С. 139.

4. Seidel J. J. Strongly regular graphs with $(-1,1,0)$ adjacency matrix having eigenvalue 3 // Lin. Alg. Appl. 1968. V. 1. № 2. P. 281-298.

5. Buekenhout F., Hubaut X. Locally polar spaces and related rank 3 groups // J. Algebra. 1977. V. 45. № 2. P. 391-434.

6. Махнёв А. А. О сильно регулярных графах с $\lambda=1$ // Матем. заметки. 1988. Т. 34. № 5 . C. $667-672$.

г. Екатеринбург, Институт математики

Поступило в редакцию и механики УрO РАH, 3.XI.1995

e-mail: mak@top.imm.intec.ru 